\title{
The Psychological Impact of COVID-19 Pandemic on Mental Health of Iranian Population
}

\author{
Seyede Momeneh Mohammadi ${ }^{1}$, Sara Ashtari ${ }^{2}$, Masoum Khosh Fetrat ${ }^{3 *}$ \\ ${ }^{1}$ Department of Anatomical Sciences, Faculty of Medicine, Zanjan University of Medical Sciences, Zanjan, Iran \\ ${ }^{2}$ Gastroenterology and Liver Diseases Research Center, Research Institute for Gastroenterology and Liver Diseases, Shahid \\ Beheshti University of Medical Sciences, Tehran, Iran \\ ${ }^{3}$ Department of Anesthesiology and Critical Care, Khatamolanbia Hospital, Zahedan University of Medical Sciences, \\ Zahedan, Iran \\ Corresponding Author: Masoum Khosh Fetrat, MD, Associate Professor, Department of Anesthesiology and Critical \\ Care, Khatamolanbia Hospital, Zahedan University of Medical Sciences, Zahedan, Iran. Tel: +98-9196017138, Email: \\ drkhoshfetrat@yahoo.com
}

Received November 5, 2020; Accepted December 6, 2020; Online Published December 8, 2020

\begin{abstract}
Introduction: As the coronavirus disease 2019 (COVID-19) pandemic rapidly sweeps across the world, it is inducing a considerable degree of psychological problems, which can affect everyone in the community. This study aimed to compare the severity of the psychological distress in the general population and patients during the COVID-19 epidemic in an Iranian population.

Methods: In this cross-sectional study, the mental health status of 221 COVID-19 infected patients and 241 participants from general population were investigated by the self-report questionnaire of Depression, Anxiety, and Stress Scale (DASS). The DASS-21 questionnaire and sociodemographic data sheet were filled out by the participants. All statistical analyses were performed using SPSS software version 21.

Results: The results indicated higher scores of DASS in patients than the population simultaneously (Wilks Lambda $=0.934, \mathrm{~F}(3$, $440)=10.44, P<0.001$ ) and individually (Mean difference $[\mathrm{MD}]=2.55,95 \% \mathrm{Cl}=1.48$ to 3.62 for depression, $\mathrm{MD}=1.48,95 \% \mathrm{Cl}=0.39$ to 2.57 for anxiety, and $\mathrm{MD}=1.41,95 \% \mathrm{Cl}=0.32$ to 2.49 for depression score).

Conclusion: The present study revealed a high prevalence of mental health problems among patients with COVID-19 and gaps in providing them with mental health services. We call for systematic screening of mental health status for all patients and developing specific psychological interventions for this vulnerable population. Psychosocial assessment and monitoring should be developed in the community to support aspects of COVID-19.

Keywords: Coronavirus Disease 2019, Psychological Distress, Stress, Anxiety, Depression, Iran
\end{abstract}

Citation: Mohammadi SM, Ashtari S, Khosh Fetrat M. The psychological impact of COVID-19 pandemic on mental health of Iranian population. Int J Travel Med Glob Health. 2021;9(1):19-24. doi:10.34172/ijtmgh.2021.04.

\begin{abstract}
Introduction
Coronavirus disease 2019 (COVID-19) caused by a new coronavirus is called severe acute respiratory syndrome coronavirus2 (SARS-CoV-2), detected in Wuhan city, China on December 31, 2019, and then the World Health Organization (WHO) declared the outbreak a global health emergency on January 30, 2020, and a global pandemic on March 11, 2020. ${ }^{1}$ The global pandemic of COVID-19 infection has emerged as a highly pathological and widespread virus in humans and as a serious disease in the field of public health and global concern with high morbidity and mortality rates. ${ }^{2}$ According to the study by Jin et al, ${ }^{3}$ about $13 \%$ of the patients with confirmed COVID-19 infection reported severe respiratory symptoms,
\end{abstract}

among which $2 \%$ died. As of 29 November 2020, a total of 62634553 confirmed cases of COVID-19 have been reported, including, 1459325 deaths and 43241504 have been reported as recovered from COVID-19 worldwide. ${ }^{4}$

Given the prevalence and mortality rate of the COVID-19 pandemic, it has affected economic, political, social, and even military sectors in all the countries around the world. Therefore, this pandemic is not just a medical phenomenon; it especially affects the mental health of individuals, which raising worries of widespread panic and growing anxiety in individuals exposed to the hazard of the virus or among certain groups in particular, older adults, care providers and people with underlying health conditions. ${ }^{5,6}$ Thus, it is highly

Copyright $\odot 2021$ The Author(s). This is an open-access article distributed under the terms of the Creative Commons Attribution License (http:// creativecommons.org/licenses/by/4.0), which permits unrestricted use, distribution, and reproduction in any medium, provided the original work is properly cited. 
important to consider the psychological effects of this viral disease on individuals' mental health at different levels of society. In a recently published study of the general population in China during the COVID-19 outbreak, people reported suffering from mental health problems with the highest percentages accounted for by stress, anxiety, and depression, which were $8.1 \%, 28.8 \%$, and $16.5 \%$, respectively. ${ }^{7}$ COVID-19 has been highlighted as a newly identified pathogen with a fast transmission pattern, absence of definitive treatment protocol or vaccination program, which has added panic, stress, anxiety, and the potential for depression among people in the community. ${ }^{8}$

Considering the rapid spread of this disease and the paucity of research in this regard, it seems necessary to do more research to help identify the disease and its related psychological factors. In particular, psychological factors, such as stress, anxiety, and tension have negatively impacted or weakened the immune system which makes it vulnerable to the diseases such as COVID-19.9,10 People need to learn appropriate strategies to tackle these traumatic factors. Therefore, predicting the expected mental and physical health impacts as well as the most susceptible populations could be valuable to develop psychosocial assessment and monitor the support aspects and effects of COVID-19. ${ }^{11}$ Here we presented the cross-sectional survey to compare the severity of the psychological distress (stress, anxiety, and depression) in general population and patients with COVID-19 in an Iranian population.

\section{Methods}

Study Design

In this comparative cross-sectional study, we measured the prevalence and compared the severity of psychological distress (stress, anxiety, and depression) in patients with COVID-19 and general population in an Iranian population. In addition, the objectives of the study were explained to all participants, and written informed consent was obtained from all. They were also assured of confidentiality.

\section{Study Population}

Of the 462 participants in this cross-sectional survey, 241 (52.2\%) were selected from the general population and 221 (47.8\%) were patients with COVID-19. Adult subjects aged 18 years or older, interested in participation, being able to read and write, and having no physical disability or mental disorder (based on self-reports) were included in the survey as a general population group. Patients with COVID-19 were selected from those referred to Baqiyatallah hospital, Tehran, Iran, which was one of the main referral centers for specialized diagnosis and treatment of COVID-19, between February and March 2020. All patients with COVID-19 enrolled in this study were diagnosed according to World Health Organization interim guidance. ${ }^{12}$ The diagnosis was made based on the chest computed tomographic (CT) scan plus positive results on a reverse transcriptase-polymerase chain reaction (RTPCR) assay of a specimen obtained on a nasopharyngeal swab indicated the confirmation of COVID-19. All patients in the study were positive based on these two methods.
Sample Size

Cochran's sample size estimation formula in the epidemiologic study was used. ${ }^{13}$ The first and second type errors were considered 0.05 and 0.02 , respectively. A $50 \%$ satisfaction probability was assumed to estimate the maximum sample size for each group (190 participants). According to the nature of the study and the probability of dropout, a $10 \%$ drop was considered and the final sample size was considered to be at least 200 participants in each group. However, more participants were willing to collaborate, hence our sample size was larger in both groups.

\section{Data Collection}

Sociodemographic characteristics data sheet, including age, sex, marital status, education, occupation, and co-morbidities was distributed and completed by all participants. In addition, the mental health status of respondents was measured using the Depression, Anxiety, and Stress Scale (DASS-21). ${ }^{14,15}$ DASS is a short screening tool that measures depression, anxiety, and stress by a 21-item self-report questionnaire. For each disorder, seven questions are considered, and the final score is obtained by the total score of the questions related to it. Each question was scored using a Likert-scale ranging from 0 (did not apply to me at all/never) to 3 (applied to me very much, or most of the time/almost always). Higher scores indicated a higher level of the disorder based on a specific classification scoring system. Individuals were categorized into normal, mild, moderate, severe, and extremely severe based on their responses. In this survey, we employed the Persian format of this questionnaire. The validity and reliability of the translated version of the Persian questionnaire were confirmed by Sahebi et $\mathrm{al}^{16}$ with a high internal correlation; Cronbach's alpha of depression, anxiety, and stress subscales were $0.77,0.79$, and 0.78 , respectively. Moreover, Moradipanah et $\mathrm{al}^{17}$ reported a Cronbach's alpha of 0.94 for depression, 0.92 for anxiety, and 0.82 for stress.

\section{Statistical Analysis}

Categorical variables were described as frequency rates and percentages, and continuous variables were described using mean \pm standard deviation (SD) values. Means for continuous variables were compared using independent group t-tests and Chi-square test or Fisher's exact test (in case of low sample) for categorical variables. The scores of the DASS subscales for each group were expressed as mean and standard deviation. To assess the effect of intervention, the analysis of multivariate analysis of variance (MANOVA) was used for controlling age, gender, and marital status variable as confounders in the main effects model. All tests were two-tailed, with a significance level of $P<0.05$. Statistical analysis was performed using SPSS software version 21 .

\section{Results}

A total of 462 participants responded to the questionnaire. Among these participants, 241 (27.2\%) were selected from general population, including 96 male (39.8\%) and 145 female $(60.2 \%)$. The mean age for the respondents in the general population was $49.16 \pm 8.01$ years. In addition, 221 
(24.9\%) questionnaires were distributed among patients with COVID-19, including 204 male (92.3\%) and 17 female (7.7\%). The mean age of patients with COVID-19 was $45.90 \pm 7.77$ years. The mean score of depression in general population was higher in females $(26.63 \pm 4.34$ vs. $25.29 \pm 4.37, P=0.020)$ and also in participants under 40 years $(27.92 \pm 5.19$ vs. $25.90 \pm 4.26, P=0.033)$ than in males and subjects over 40 years. Occupational status for the general population and patients with COVID-19 was divided into five subgroups: employed, self-employed, job seeker, or retiree, student, and housewife. The highest frequency in both groups was relevant to the self-employment subgroup (more than 35\%). However, this factor did not affect stress, anxiety, and depression in these two groups. In addition, there was no significant difference between the two groups in terms of education and co-morbidities and also the level of education did not affect psychological distress. However, the mean score of anxiety in patients with COVID-19 was significantly higher in patients with comorbidity diseases compared to patients without any comorbidity $(28.14 \pm 5.07$ vs. $26.68 \pm 5011, P=0.043)$. Table 1 shows the sociodemographic characteristics and severity of psychological distress in participants in the two study groups.

In terms of severity of psychological distress, the results showed that the majority of participants in the general population and patients with COVID-19 had extremely severe anxiety, 231 (95.9\%) and 215 (97.5\%), respectively. Nearly half of the participants in both groups had severe stress; 118 (49\%) participants in the general population and 103 (46.6\%) patients with COVID-19. In terms of depression, half of the participants in the general population had severe depression $(n=124,51.5 \%)$, while more than half of patients with COVID-19 had extremely severe depression $(n=120$, $54.3 \%)$.

The comparisons of the mean score of the depression,

Table 1. Sociodemographic Characteristics and Severity of Psychological Distress in the 2 Study Groups

\begin{tabular}{|c|c|c|c|c|}
\hline Variables & & Community Population $(n=241)$ & Patients With COVID-19 $(n=221)$ & $P$ Value \\
\hline Age & $($ Mean \pm SD $)$ & $49.16 \pm 8.01$ & $45.90 \pm 7.77$ & $<0.001$ \\
\hline \multirow{2}{*}{ Gender } & Male $(\%)$ & $96(39.8)$ & $204(92.3)$ & $<0.001$ \\
\hline & Female (\%) & $145(60.2)$ & $17(7.7)$ & \\
\hline \multirow{2}{*}{ Marital status } & Married (\%) & $151(62.7)$ & $99(44.8)$ & $<0.001$ \\
\hline & Unmarried (\%) & $90(37.3)$ & $122(55.2)$ & \\
\hline \multirow{5}{*}{ Occupation } & Employed & $31(12.9)$ & $34(15.4)$ & 0.787 \\
\hline & Self-employed & $82(34)$ & $82(37.1)$ & \\
\hline & Out of work or retired & $64(26.6)$ & $54(24.4)$ & \\
\hline & Student & $49(20.3)$ & $39(17.6)$ & \\
\hline & Housewife & $15(6.2)$ & $12(5.4)$ & \\
\hline \multirow{6}{*}{ Education } & High School & $4(1.7)$ & $6(2.7)$ & 0.834 \\
\hline & Diploma & $22(9.1)$ & $24(10.9)$ & \\
\hline & Associate & $59(24.5)$ & $65(29.4)$ & \\
\hline & Bachelor & $78(32.4)$ & $69(31.2)$ & \\
\hline & Master degree & $60(24.9)$ & $44(19.9)$ & \\
\hline & $\mathrm{PhD}$ & $18(7.5)$ & $13(5.9)$ & \\
\hline \multirow{7}{*}{ Comorbidities } & Non & $158(65.6)$ & $144(65.2)$ & 0.876 \\
\hline & Cardiovascular & $10(4.1)$ & $16(7.2)$ & \\
\hline & Diabetes & $16(6.6)$ & $21(9.5)$ & \\
\hline & Hypertension & $21(8.7)$ & $16(7.2)$ & \\
\hline & Allergy & $20(8.3)$ & $12(5.4)$ & \\
\hline & Chronic kidney disease & $9(3.7)$ & $7(3.2)$ & \\
\hline & Chronic liver disease & $7(2.9)$ & $5(2.3)$ & \\
\hline \multirow{5}{*}{ Stress } & $($ Mean \pm SD $)$ & $27.34 \pm 4.37$ & $28.59 \pm 5.18$ & 0.005 \\
\hline & Mild & $5(2.1)$ & $1(0.5)$ & \\
\hline & Moderate & $106(44)$ & $94(42.5)$ & \\
\hline & Severe & $118(49)$ & $103(46.6)$ & \\
\hline & Extremely severe & $12(5)$ & $23(10.4)$ & \\
\hline \multirow{3}{*}{ Anxiety } & $($ Mean \pm SD $)$ & $26.04 \pm 4.52$ & $27.62 \pm 5.12$ & $<0.001$ \\
\hline & Severe & $10(4.1)$ & $6(2.7)$ & \\
\hline & Extremely severe & $231(95.9)$ & $215(97.3)$ & \\
\hline \multirow{4}{*}{ Depression } & $($ Mean $\pm \mathrm{SD})$ & $26.09 \pm 4.39$ & $28.07 \pm 5.06$ & $<0.001$ \\
\hline & Moderate & $25(10.4)$ & $16(7.2)$ & \\
\hline & Severe & $124(51.5)$ & $85(38.5)$ & \\
\hline & Extremely severe & $92(38.2)$ & $120(54.3)$ & \\
\hline
\end{tabular}


anxiety, and stress between the two groups of study are presented in Figure 1. The mean scores of stress, anxiety, and depression were compared between the two groups of study by $t$ test. Results showed that the mean scores of stress ( $28.59 \pm 5.18$ vs. $27.34 \pm 4.37, P=0.005)$, anxiety $(27.62 \pm 5.12$ vs. $26.04 \pm 4.52, P<0.001)$, and depression $(28.07 \pm 5.06$ vs. $26.09 \pm 4.39, P<0.001)$ in patients with COVID-19 were significantly higher than those in the general population. In addition, MANOVA test was used to compare the mean scores of psychological distress between two groups with adjusted sociodemographic characteristic, including gender, marital status, occupation, education, co-morbidities, and age whose results are presented in Table 2. The results of this study showed higher scores of DASS among patients group than the population (Wilks' lambda $=0.934, \mathrm{~F}_{(3,440)}=10.44$, $P<0.001$ ) and individually (Mean difference $[\mathrm{MD}]=2.55$, $95 \% \mathrm{CI}=1.48$ to 3.62 for depression, $\mathrm{MD}=1.48,95 \% \mathrm{CI}=$ 0.39 to 2.57 for anxiety, and $\mathrm{MD}=1.41,95 \% \mathrm{CI}=0.32$ to 2.49 for depression score). After adjusting gender, age, occupation type, education level, and having co-morbidities, the results remained significant (all $P<0.05$ ).

\section{Discussion}

The current COVID-19 outburst caused fear on a social level which caused psychological disorders, including depression, anxiety, and stress. The results of the present study and all previous studies show that the psychological trauma caused by the prevalence of infectious diseases is highly common in societies. ${ }^{18-20}$ Infectious pandemics can cause disruptions in societies and individuals on many levels. COVID-19 imposes irreversible psychological impacts on all groups of community members due to the commuting restrictions, fear of contracting the virus, anxiety about the closure of schools and businesses, the depression following the loss of friends and family, and fear of death. ${ }^{21-23}$

The main results of the present study showed that COVID-19 patients had significantly higher scores of stress, anxiety, and depression compared to the general population; this suggests that they, as the main target group most affected by the psychological problems of the disease, need more support and more psychiatric care. In a total of 462 participants in this survey, the anxiety score was higher in men than in women. In addition, the score of depression in females and also in participants under 40 years among the general population group was significantly higher than the males and subjects over 40 years. The analysis of the other sociodemographic characteristics such as marital status,

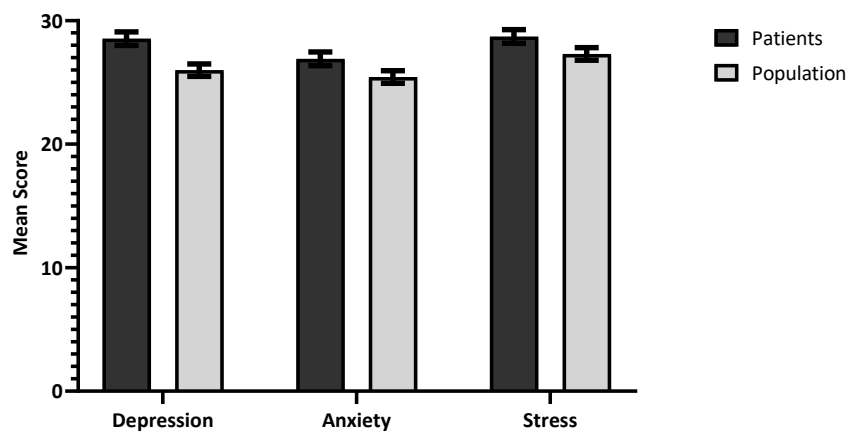

Figure 1. The Mean Score of the Depression, Anxiety, and Stress in Patients With COVID-19 and Population.

education level, and the occupation of participants in both groups did not show any significant effects on the scores of psychological distress. However, the mean score of anxiety in patients with COVID-19 was significantly higher in patients with comorbidity diseases compared to patients without any comorbidity.

Unpredictability, uncertainty, the seriousness of the disease, misinformation, social isolation, and the overwhelming news may cause anxiety and fear in the public. The general public may also experience frustration, and irritability under isolation measures. ${ }^{24}$ According to the results of our study, the psychological effects of COVID-19 as depression was higher in women than those in men among general population, which was consistent with the results of previous studies in China $^{6,25}$ which showed that the women compared to men had more experienced psychological symptoms related to this pandemic. Because of the pandemic and this exceptional situation such as lockdown and quarantine, the life condition of families suddenly and deeply changed. In the home environment, the educational role of parents for children has become even much crucial than before. Thus, mothers are faced with additional responsibilities that can significantly increase the risk of experiencing stress and negative emotions in females. ${ }^{26,27}$ Hence, supporting women in this situation might be especially important. In addition, extensive epidemiological studies have shown that women are at higher risk for depression than men. ${ }^{28}$ On the other hand, the results showed that the score of anxiety level in men was significantly higher than that in women. This could be attributed to economic pressures during the pandemic which affects most men rather than women. Hence, economic pressure can put men under psychological pressure.

In patients with COVID-19, these can be relevant to the

Table 2. Comparison of Depression, Anxiety, and Stress in Patients and Population Simultaneously

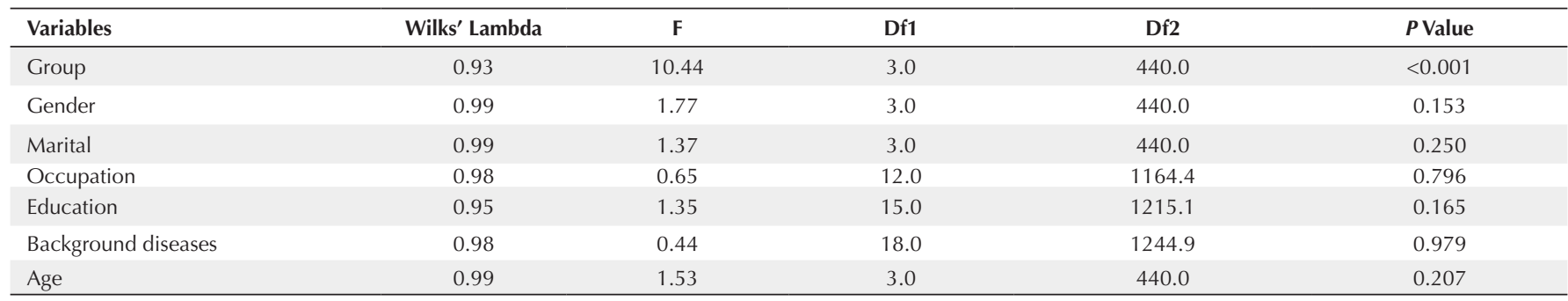

MANOVA adjusted for gender, marital, occupation, education level, comorbidity disease and age. 


\section{Research Highlights}

\section{What Is Already Known?}

The study showed a high severity of anxiety, stress and depression among Iranian populations during the pandemic of COVID-19.

\section{What This Study Adds?}

The present study revealed a high prevalence of mental health problems among patients with COVID-19 and the gaps in mental health services for them. Therefore, psychosocial assessment and monitoring should be developed in the community to support the aspects of COVID-19.

fear of severe disease consequences, contagion, isolation treatment, loss of trust in health services, and fear of death. Consequently, they may experience loneliness, denial, anxiety, depression, insomnia, and despair, which may lower treatment adherence. Moreover, previous studies during the outbreak of severe acute respiratory syndrome (SARS), and Middle-East respiratory syndrome (MERS) have shown that depression and post-traumatic stress disorders (PTSDs) persist for up to one year after illness. ${ }^{29-31}$ Therefore, we should expect long-term negative psychological outcomes as PTSD among COVID-19 survivors and general population. ${ }^{32}$ As a possible solution for this challenge, public health decision-makers need to perform appropriate psychosocial interventions and incorporate mental health management plans. Other steps to lower the psychological distress in society can be the assessment of the accuracy of the information, enhancing social support, reducing the stigma associated with the disease, maintaining a normal life while adhering to safety measures, and using available psychosocial services ${ }^{8}$; therefore, a need of comprehensive infection control practices are necessitated to help patients, families, and society to deal with the threat. ${ }^{25}$ There are some limitations to the current study. First, this is a cross-sectional study for rapid assessment for stress, anxiety, and depression during the pandemic between two groups of general population and patients with COVID-19, and we are unable to investigate causal relationships. Second, the convenient sampling method for general population group and self-reported questionnaire could lead to highly vulnerable to selection bias and high level of sampling error. Third, we were unable to investigate the history of participants' mental disorders. Thus, participants with a history of mental disorders based on self-report were excluded from the study.

\section{Conclusion}

In conclusion, this study showed the high severity of anxiety, stress, and depression among general population and patients with COVID-19 during the pandemic. The patients with COVID-19 are the main targets of psychiatric assessment and care. Increasing public awareness of the disease, building trust in the media, and providing information on patients' recovery can reduce psychological distress in society. Therefore, designing psychological interventions is essential to improve mental health during and after the pandemic.

\section{Authors' Contributions}

MKF developed the study concept. All authors contributed to the study design. Testing and data collection were performed by MKF and SMM. Data analysis and interpretation was performed by MKF, SA, and SMM. All authors approved the final version of the manuscript for submission.

\section{Conflict of Interest Disclosures}

The authors report no known conflict of interest.

\section{Ethics Approval}

This study was approved by the Baqiyatallah University of Medical Sciences Ethics Committee, Tehran, Iran, with the code of IR.BMSU.REC.1398.438. Informed consent was taken from all participants.

\section{Funding/Support}

This research did not receive any specific grant from funding agencies in the public, commercial, or not-for-profit sectors.

\section{References}

1. World Health Organization (WHO). Coronavirus Disease 2019 (COVID-19): Situation Report, 51. WHO; 2020. https://cutt.ly/ qtW0ZpO. Updated March 12, 2020. Cited March 12, 2020.

2. Lai CC, Shih TP, Ko WC, Tang HJ, Hsueh PR. Severe acute respiratory syndrome coronavirus 2 (SARS-CoV-2) and coronavirus disease-2019 (COVID-19): the epidemic and the challenges. Int J Antimicrob Agents. 2020;55(3):105924. doi:10.1016/j. ijantimicag.2020.105924.

3. Jin $\mathrm{YH}$, Cai L, Cheng ZS, et al. A rapid advice guideline for the diagnosis and treatment of 2019 novel coronavirus (2019-nCoV) infected pneumonia (standard version). Mil Med Res. 2020;7(1):4. doi:10.1186/s40779-020-0233-6.

4. Worldometer: COVID-19 Coronavirus Outbreak. https://www. worldometers.info/coronavirus/. Accessed April 4, 2020.

5. Xiang YT, Yang Y, Li W, et al. Timely mental health care for the 2019 novel coronavirus outbreak is urgently needed. Lancet Psychiatry. 2020;7(3):228-229.doi:10.1016/s2215-0366(20)30046-8.

6. Wang C, Pan R, Wan X, et al. Immediate psychological responses and associated factors during the initial stage of the 2019 coronavirus disease (COVID-19) epidemic among the general population in China. Int J Environ Res Public Health. 2020; 17(5):1729. doi:10.3390/ijerph17051729.

7. Wang C, Pan R, Wan X, et al. A longitudinal study on the mental health of general population during the COVID-19 epidemic in China. Brain Behav Immun. 2020;87:40-48. doi:10.1016/j. bbi.2020.04.028.

8. World Health Organization (WHO). Mental Health and Psychosocial Considerations During the COVID-19 Outbreak. https://www.who.int/docs/default-source/coronaviruse/mentalhealth-considerations.pdf. Published March 18, 2020.

9. Segerstrom SC, Miller GE. Psychological stress and the human immune system: a meta-analytic study of 30 years of inquiry. Psychol Bull. 2004;130(4):601-630. doi:10.1037/00332909.130.4.601

10. Mattos Dos Santos R. Isolation, social stress, low socioeconomic status and its relationship to immune response in Covid-19 pandemic context. Brain Behav Immun Health. 2020;7:100103. doi:10.1016/j.bbih.2020.100103

11. Mason BW, Lyons RA. Acute psychological effects of suspected bioterrorism. J Epidemiol Community Health. 2003;57(5):353- 
354. doi:10.1136/jech.57.5.353.

12. World Health Organization (WHO). Clinical Management of Severe Acute Respiratory Infection when Novel Coronavirus (2019nCoV) Infection is Suspected: Interim Guidance. WHO; 2020. https://www.who.int/publications-detail/clinical-managementofsevere-acute-respiratory-infection-when-novelcoronavirus-(ncov)infection-is-suspected. Accessed January 31, 2020. Published 2020.

13. Cochran WG. Sampling Techniques. 2nd ed. New York: John Wiley \& Sons; 1963.

14. Lovibond PF. Long-term stability of depression, anxiety, and stress syndromes. J Abnorm Psychol. 1998;107(3):520-526. doi:10.1037//0021-843x.107.3.520.

15. Norton PJ. Depression Anxiety and Stress Scales (DASS-21): psychometric analysis across four racial groups. Anxiety Stress Coping. 2007;20(3):253-265. doi:10.1080/10615800701309279.

16. Sahebi A, Asghari MJ, Salari RS. Validation of Depression Anxiety and Stress Scale (DASS-21) for an Iranian population. Journal of Iranian Psychologists. 2005;1(4):299-313. [Persian].

17. Moradipanah F, Mohammadi E, Mohammadil AZ. Effect of music on anxiety, stress, and depression levels in patients undergoing coronary angiography. East Mediterr Health J. 2009;15(3):639647. doi:10.26719/2009.15.3.639.

18. McAlonan GM, Lee AM, Cheung V, et al. Immediate and sustained psychological impact of an emerging infectious disease outbreak on health care workers. Can J Psychiatry. 2007;52(4):241-247. doi:10.1177/070674370705200406.

19. Tucci V, Moukaddam N, Meadows J, Shah S, Galwankar SC, Kapur GB. The forgotten plague: psychiatric manifestations of Ebola, Zika, and emerging infectious diseases. J Glob Infect Dis. 2017; 9(4):151-156. doi:10.4103/jgid.jgid_66_17.

20. AINajjar NS, Attar LM, Farahat FM, AlThaqafi A. Psychobehavioural responses to the 2014 Middle East respiratory syndrome-novel corona virus (MERS CoV) among adults in two shopping malls in Jeddah, western Saudi Arabia. East Mediterr Health J. 2017; 22(11):817-823. doi:10.26719/2016.22.11.817.

21. Kamal NM, Othman N. Depression, anxiety, and stress in the time of COVID-19 pandemic in Kurdistan Region, Iraq. Kurd J Appl Res. 2020. doi:10.24017/covid.5.

22. Odriozola-González P, Planchuelo-Gómez Á, Irurtia MJ, de Luis-García R. Psychological effects of the COVID-19 outbreak and lockdown among students and workers of a Spanish university. Psychiatry Res. 2020;290:113108. doi:10.1016/j. psychres.2020.113108.

23. Mazza C, Ricci E, Biondi S, et al. A nationwide survey of psychological distress among Italian people during the COVID-19 pandemic: immediate psychological responses and associated factors. Int J Environ Res Public Health. 2020;17(9):3165. doi:10.3390/ijerph17093165.

24. Li W, Yang Y, Liu ZH, et al. Progression of mental health services during the COVID-19 outbreak in China. Int J Biol Sci. 2020; 16(10):1732-1738. doi:10.7150/ijbs.45120.

25. Liu D, Ren Y, Yan F, et al. Psychological Impact and Predisposing Factors of the Coronavirus Disease 2019 (COVID-19) Pandemic on General Public in China. SSRN Electronic Journal. 2020. doi:10.2139/ssrn.3551415.

26. Vahedian-Azimi A, Moayed MS, Rahimibashar F, Shojaei S, Ashtari S, Pourhoseingholi MA. Comparison of the severity of psychological distress among four groups of an Iranian population regarding COVID-19 pandemic. BMC Psychiatry. 2020;20(1):402. doi:10.1186/s12888-020-02804-9.

27. Spinelli $M$, Lionetti F, Pastore $M$, Fasolo $M$. Parents' stress and children's psychological problems in families facing the COVID-19 outbreak in Italy. Front Psychol. 2020;11:1713. doi:10.3389/ fpsyg.2020.01713.

28. Lim GY, Tam WW, Lu Y, Ho CS, Zhang MW, Ho RC. Prevalence of depression in the community from 30 countries between 1994 and 2014. Sci Rep. 2018;8(1):2861. doi:10.1038/s41598-01821243-x.

29. Lee AM, Wong JG, McAlonan GM, et al. Stress and psychological distressamongSARSsurvivors 1 yearaftertheoutbreak. CanJPsychiatry. 2007;52(4):233-240.doi:10.1177/070674370705200405.

30. Tansey CM, Louie M, Loeb M, et al. One-year outcomes and health care utilization in survivors of severe acute respiratory syndrome. Arch Intern Med. 2007;167(12):1312-1320. doi:10.1001/ archinte.167.12.1312.

31. Moldofsky H, Patcai J. Chronic widespread musculoskeletal pain, fatigue, depression and disordered sleep in chronic post-SARS syndrome; a case-controlled study. BMC Neurol. 2011;11:37. doi:10.1186/1471-2377-11-37.

32. Lee SM, Kang WS, Cho AR, Kim T, Park JK. Psychological impact of the 2015 MERS outbreak on hospital workers and quarantined hemodialysis patients. Compr Psychiatry. 2018;87:123-127. doi:10.1016/j.comppsych.2018.10.003. 